\title{
Contact Principles and TCM`S Management on Post-Syndromes of Covid-19
}

\author{
Dan Jiang MMedSci, FBAcC, FATCM \\ Hallam Institute of TCM, 439 Glossop Road Sheffield S10 2PR UK.
}

*Corresponding Author: Dan Jiang, Hallam Institute of TCM, 439 Glossop Road Sheffield S10 2PR UK.

\section{Received Date: 31 August 2021 | Accepted Date: 30 September 2021 | Published Date: 05 October 2021}

Citation: D Jiang. (2021) Contact Principles and TCM`S Management on Post-Syndromes of Covid-19. J. Clinical and Laboratory Research. 3(4); DOI:10.31579/2768-0487/046

Copyright: @2021 Dan Jiang. This is an open-access article distributed under the terms of the Creative Commons Attribution License, which permits unrestricted use, distribution, and reproduction in any medium, provided the original author and source are credited.

\begin{abstract}
:
The coronavirus disease 2019 (COVID-19) has still been spreading around the world, causing an outbreak of acute infectious pneumonia, even a multiple systematic inflammatory disorders following with varied virus. From World Health Organizations (WHO) weekly report in $16^{\text {th }}$ Aug 2021, more than 2 hundred millions (206 693 357) of infected cases and more than 4 million (4 352488 ) of deaths are confirmed in the world [1]. The daily report In UK in $18^{\text {th }}$ Aug 2021, there are more than 6 million of infected cases (6 295 613) and 130 thousands of died cases (130 979) are confirmed, fluctuating figures on daily recently on infected and died cases with Covid-19 are reported although the severe case rates, hospitalized rates and died rate have decreased in UK [2]

Due to such bigger amount of patients' appearance, some post-syndrome of Covid-19 are unavoidably leaving after they are self-healed or treated in the hospitals or anywhere else, they have not recovered completely, still remain some unwanted symptoms, some of them can be quite serious which are possible to disturb them for the rest of their life. We should do and be able doing some significant helps for them with Chinese herbal medicine (TCM). I will discuss these common occurrences of post- syndromes which I have treated: inferior function of lungs, dysfunction of gastrointestinal system, Psychiatric disorder, Post-virus-Chronic-fatigue syndrome, post inflammation of Sexual organs and how manage these post syndromes of Covid-19 with TCM here. TCM should play more effect for helping postsyndromes of Covid-19 after reopening clinics with full of our treating technologies.

Key words: Covid-19; post syndrome of Covid-19; TCM
\end{abstract}

\section{Introduction:}

The coronavirus disease 2019 (COVID-19) has still been spreading around the world, causing an outbreak of acute infectious pneumonia, even a multiple systematic inflammatory disorders following with varied virus. From World Health Organizations (WHO) weekly report in $16^{\text {th }}$ Aug 2021, more than 2 hundred millions (206 693 357) of infected cases and more than 4 million (4 352488 ) of deaths are confirmed in the world [1]. The daily report In UK in $18^{\text {th }}$ Aug 2021, there are more than 6 million of infected cases (6 295 613) and 130 thousands of died cases (130 979) are confirmed, fluctuating figures on daily recently on infected and died cases with Covid-19 are reported although the severe case rates, hospitalized rates and died rate have decreased in UK [2].

China, as the earliest country in which Covid-19 was erupted can quickly and effectively control it's spreading in less than 2 months, the Chinese herbal medicine demonstrates a unique and excellent effect, and play an important role for them in there. Due to TCM is involved in the main medical stream in China, so TCM doctors can manage herbal medicines for patients with Covid-19 in the minor and ordinary stages when they are admitted in the temporary hospitals for changing their positive PCR into negative; they can be consulted with conventional medical doctors together in the hospitals for intervening to rescue and treat to the patients with Covid-19 in the serious and critical states for making the cured rates increasing [3]. So the authority of China issues the national treating guideline for Covid-19 to recommend Chinese herbal medicine for Covid19 which should be used as early as possible [4].

In UK and most of West countries, we do Traditional Chinese Medicine (TCM) as complementary and alternative medicine (CAM) out of the main stream--National health service (NHS), due to lack of sufficient preventive facilities and equipment in the TCM clinics as private institution, we are required to close during the lockdown phase. But I can contact many cases as a volunteer TCM consultant invited by the Oversea Chinese TCM Forum for introducing to Chinese and students in UK, and some of my old patients and relations, so more and more requires daily from WeChart, What'sup, Internet, Message and these uncontacted accesses come to me. I collect clinical information with TCM's diagnostic 
model from them and arrange relevant herbal medicines to post to them. I have been contacting more than hundreds of requires since last March, among of them, many peoples may be infected or suspected patients with Covid-19 [5].

Following with gradual social reopening UK, when the clinics of CAM will be facing to reopen, we have to think how manage such more patients with Covid-19 who are still leaving post-syndromes after they are selfhealed or treated in the NHS or anywhere else, they have not recovered completely, still leave some unwanted symptoms which are possible to disturb them for the rest of their life. We should do and be able doing some significant helps for them. I will discuss these common occurrences of post- syndromes and how to manage the post syndromes of Covid-19 with TCM here.

\section{Primary communications with requires in an uncontacted access:}

For double care to prevent for infecting to the practitioner and staffs, and contaminating to the clinic, we should build an uncontacted access: all of patients in either the old patients or new visitors should be contacted with practitioners in Whatsapp, WeChart, Phone or internet and any uncontacted access (AUA) before visiting to the clinic, we should know general condition on him/her for identifying her/ his contacted and suffered history, current symptoms, then we can decide whether we can give an appointment for him/her, or not?

We can accept the old patients who have been confirmed no any Covid19 contacted and suffered history for making an appointment; but for some ones who confuse whether (s) he contacts or suffers from Covid-19, or has suffered Covid-19 should be identified whether he or she is able to come to the clinic for his /her treatment in a safe way?

All of old patients or new visitors should fill the form---Health information on Covid-19 consent form issued by BAcC [6] before (s) he is accepted for treatment.

\section{Identify whether (s) he was a sufferer with Covid-19 and accepted principles:}

We can have these 5 questions for identifying to his/her state on Covid19 ?

Did (s) he had an exposed history to patients with Covid-19? Such as a sufferer who is diagnosed as Covid-19 in family members, colleagues, close friends, neighbors etc.; or did (s) he take a public transportations for work or live, such as underground, bus, train, airplane etc.; or did (s) he go to supermarket, superstore, shop Centre, public game, cinema, theater etc. where is possible to contact or close to sufferers.

If these is a positive contact history, we can think/ suspect that (s) he might be a sufferer with Covid-19?

Has (s) he been diagnosed as Covid-19? What kind of treatment (s) he has accepted? Whether was he /she admitted in hospital?

If there is a positive diagnostic and treating procedure, we can confirm that he/she was a sufferer as Covid-19; and how severity of covid-19 was (s) he ?

Whether was he/she done a PCR test? Whether positive or negative was it? When was he/she done PCR last time, positive or not?

If (s) he was positive to PCR but negative transferred, (s) he was a sufferer; but if (s) he is still positive result on PCR, (s) he should be an active sufferer with covid-19.

Is sufferer still manifesting some symptoms although (s) he is confirmed a negative PCR, we need identifying whether he /she is a post-Covid-19 sufferer.
Whether is sufferer appearing a sign on "Pandemic dampness" tongue for differentiating whether he/she is suitable to be treated for Covid-19 by TCM.

\section{Accepted principles:}

From all of these questions, we can know whether this person has a contact history on Convid-19, whether (s) he was a sufferer, whether is (s) he is still an active patient, whether ( $\mathrm{s}$ ) he is still possessing many figures and whether is (s) he qualified to be treated by TCM?

If (s) he is an suspected sufferer, even still be an active sufferer, we should not accept her /him as a visitor to the clinic, but should communicate her/him with an uncontacted access for arranging her/his treatment;

If (s) he confuses whether (s) he is a sufferer on Covid-19 or not? Even confuses whether (s) he has got any contacted history? We should make a proper diagnosis on Covid-19 to her/ him at first, then decide whether arrange her/his to visit to the clinic;

If (s) he is confirmed no any active signs, (s) he should be identify whether a sufferer with post syndrome on Covid-19 is?

For all of them who have healed, no infected or no suspected from Covid19 , we can allow (s) he visits to the clinic for gaining an appointment and accepting our service.

\section{Identify whether (s) he is suffering from a post syndrome on Covid-19:}

The post syndrome on Covid-19 indicates to still be existing some clinical figures for disturbing the quality of life when (s) he has transferred PCR to negative and normal to the relevant examinational items after suffered from Covid-19 without life threatening.

As post-syndrome of Covid-19, it should be manifested as:

A negative PCR;

Some clinical symptoms which are caused from Covid-19, such as breathless, chest pain, tiredness, fatigue, or difficult to walk, $\mathrm{SaO} 2<95$ are produced by weaker, or collapse of lung(s) or other organs from a sufferer of Covid-19;

Some clinical symptoms which are produced along Covid-19 as complications although it is not directly caused by Covid-19; such as anxiety, depression and symptoms are still leaving to the sufferer after (s) he is healed from Covid-19;

Some clinical symptoms which are occurred related with Covid-19, such as, as a family member of sufferer, a friend or career of sufferers, (s) he feels exhaustion, fatigue, restlessness or nervousness, but no positive PCR or special symptoms of Covid-19, even no tongue sign on Pandemic dampness with TCM's perspective.

Other clinical symptom which may be related with Covid-19.

\section{Some cases of Post-syndromes of Covid-19:}

I have been contacting many cases with post-syndrome of Covid-19 along of these 6 months, and will be possible to have some more cases when we are reopening the clinic, so I will share a few of common cases here for discussing how manage them well with TCM approach.

\section{Case1: Collapse of lungs after a covid-19 suffered:}

Mr W 71 years old of retired engineer, was diagnosed as Covid-19 due to fever, cough, breathless and chest pain when his couple visited their daughter's family in London on this March; he was admitted into ICU in hospital since he manifested a difficult breaths, wheezing, even dyspnoea, dropping down to 83 in Soa2; he was discharged from hospital after a week of intensive care with oxygen inbreathing by CPAP, antibiotics and 
other relevant treatments there, he was still feeling hard to breathe through, wheezing, cough and tenderness of chest while he was a bit of removing; difficult to inbreathe while he was sleep with opening mouth and bad dream; all of symptoms aggravated if he had activity who couldn't walk in only 10 meters indoor. His SoA2 lingered around 89-91, but always worse at the left wrist than right one.......red and plump tongue with teeth marks and greasy-dry-thick coating.

Diagnosis: Post-syndrome of Covid-19 with collapses of lungs and heart

Differentiation of syndromes: Qi and Yin deficiency with unclear heat and dampness accumulation

Treating principle: Reinforcing Qi and nourishing Yin to lungs and heart; Releasing heat and expelling dampness.

Herbal prescription:

Jiangbanxia15, Chenpi10, Fuling10, Huanngqin10,

Daqingye10, Xuanshen10, Beishashen10, Maidong10,

Chuanlianzi10, Gualouren10, Baihe10, Taizishen30,

Gancao5

Above herbs was decocted as an herbal juice, twice daily, a dose for a day.

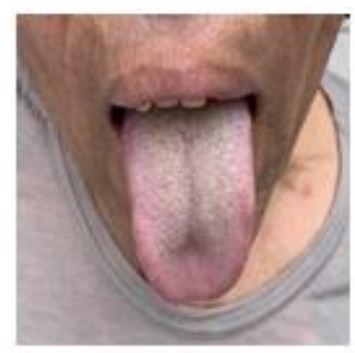

Image 1

\section{Result and Analysis:}

After the treatment with above modified herbal prescription, he felt progress week by week for a month, he can walk well indoor, and walk outside daily who was able to go up steps for 4 floors flat they was living; his SoA2 had up to 94-95. He is now recovering.

This case is leaving a post- syndrome of Covid-19, lung collapse with heart involved, since Pneumonia infected by Covid-19 in serious-critical stage. After rescuing and intensive treatment in ICU for a week, he is still staying incompletely healed for Covid-19, although he is discharged. $\mathrm{He}$ is still lower on his SoA2 which indicates the inferior function of breathing exchange in lungs; the SoA2 in left wrist lower than right which indicates an insufficiency oxygen supply on cardiological muscular. So we use the herbs for releasing the excessive heat and expelling remaining dampness to keep eliminating the rest of Coronavirus in lungs; and herbs for reinforcing Qi and nourishing Yin to promote self-healing procedure to lungs and heart for preventing these organs fibrosis' occurrence.

\section{Case 2, Inferior function of gastrointestinal system:}

Ms Li, 42 years old of house wife in London who looked for requiring to TCM consultant for her fever, cough, and breathless in the next day when her husband is admitted into ICU in hospital as Covid-19. She had 38.3C of temperature, breathless, cough, tenderness of chest; nausea, upset stomach, anorexia; and nervous, restlessness, insomnia; pale tongue with teeth marks and white greasy coating. (Tongue photo at left) After a pandemic dampness was identified in a cold and dampness pattern, she was give Huoxiangzhengqi decoction, modified with Wendan decoction for treatment. She had a bit of loose bowel at initial, gradually better after two weeks, she had a clear and less greasy coating since, she was checked immune examination who was demonstrated immunized for Covid-19 with higher IgE.

She was still manifesting discomfort in the stomach, upset easily, nausea, reflex; also grief, sorrow, gravamen, anxiety, nervous, restless after her husband was died in hospital; pale tongue with teeth marks and less-thin coating. (Tongue photo at right)

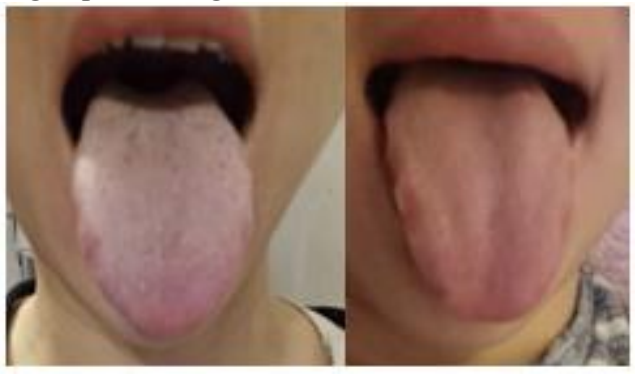

Image 2

Diagnosis: Post-syndrome of Covid-19, IBS

Differentiation of syndrome: Spleen deficiency and Liver Qi stagnation; Unharmony of stomach

Treating Principle: Strengthening spleen and harmonizing middle burner; Dredging liver and promoting Qi's remove through

Prescription: Buzhongyiqi decoction modified with Xiaoyao Wan

Huangqi 15, Dangshen10, Baizhu10, Chenpi10,

Fuling10, Shengma6, Chaihu10, Xiangfu10,

Sahren10, Ganjiang10, Danggui10, Yuanhu10,

Zhigancao5

Above herbs are decocted as herbal juice, twice daily, a dose for a day.

\section{Result and Analysis:}

This patient may have irritable bowel syndrome in the past, so she has been a spleen deficiency at basic body. She quickly manifests some symptoms and signs as a pandemic cold pattern after she is infected as coronavirus. When a relevant herbal prescription is given, she is controlled in two weeks. But she is leaving many symptoms and hard to recover under of covid-19 infected and died of husband; She has been healed after she is given treatment with strengthening qi and harmony of stomach.

\section{Case 3 Psychiatric disorder:}

Due to meeting this serious widespread on this pandemic infectious diseases, some people who possess a weaker or sensitive mind are easily affected psychiatric disorder or triggered out her /his old problem, such as Anxiety, Phobia, Depression, Sorrow etc. are common states which can be explored from an infected covid-19, or appeared just while (s) he is watching or thinking some ones else are / is infected, then (s) he manifests out a psychiatric disorder. These are the commonest clinical condition when I manage requirements.

Mrs L 42 years old of clerk, who manifests anxiety, anxious, restless, nervous, palpitation, since her husband is suspected as a covid-19 in a minor state; she has panic attacks, annoyed and weepy who worries her husband suffering from Covid-19 too severe to be died; she worries she is infected, her children are infected as well? Anxiety attacks, nervous daily until insomnia, tiredness....... When she requires for her husband's 
covid-19, her husband reports she is much serious case who needs a treatment.

She manifests that there are a light red tongue with thin white coating, I believe she doesn't have pandemic dampness as her tongue's appearance, and can't consider Covid-19 to be suffered, and a psychiatric disorder should be the main health problem for her.

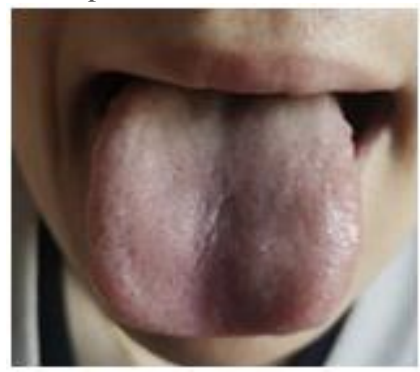

Image 3

Diagnosis: Anxiety attacks

Differentiation of syndrome: Liver qi stagnation Dampness engaging at spleen and heart

Prescription: Chaihushugan decoction variation

Chaihu10, Jiangbanxia15, Chenpi10, Fuling10,

Xiangfu10, Zhiqiao10, Huangqin10, Chishao10,

Shichangpu10, Yujin10, Cangzhu10, Yiyiren30,

Chunalianzi10, Gancao5

Above herbs are decocted as herbal juice, twice daily, a dose for a day. After she has been better in a week, she is changed to patent herbal pills, Chaihushugan Pills and Renshenguipi Pills until her recovered.

\section{Result and Analysis:}

This lady may never suffer from Covid-19, her husband looks like an infected case, but in a minor state; when the pandemic infectious disease is wide spreading throughout world, family people may be involved in, so she collapses in her mind who manifests a psychiatric disorder. She is the right case who should accept TCM treatment which should display a treating priority. When we reopen the clinic, we will make more accurate, effective result if we can plus pulse diagnosis and acupuncture treatment for supporting more and more patients in this disorders.

\section{Case 4, Post-virus and Chronic fatigue syndrome/ ME:}

Chronic fatigue syndrome is caused by a virus infection when (s) he meets a low state of her/ his constitution at initial; during this pandemic coronavirus wide spread, the sufferers will be infected by this virus, or triggered by this virus, (s) he is leaving a chronic fatigue syndrome. Sufferers with CFS / ME who can be become a negative PCR from a positive one, or without positive PCR should be good managed by TCM approach.

Miss S, 32 years old of manager suffers from a fatigue, headache, and general aching for three months. On this Feb, two colleagues of her work in same office are reported to be infected as Covid-19, although she has not been out since during the Lockdown phase, but she is always feeling tiredness, headache, general aching and discomfort; but no fever occurrence, so she believes that she needn't go to GP or hospital for requiring treatment. But she is aggravated day by day, until she feels too illness to go a shopping who has to take a rolling pulley for helping her walk. She requires with me in the internet when she knows some benefits get from TCM for her friend. Pale tongue with less-thin-white coating.

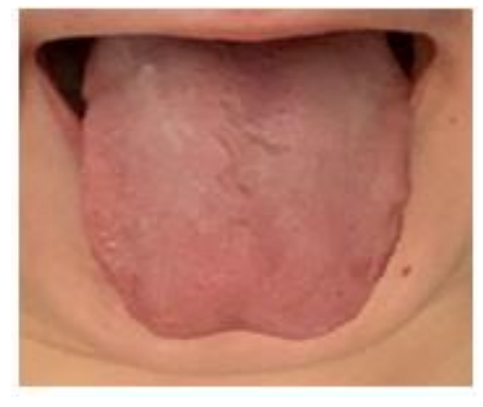

Image 4

Diagnosis: Chronic fatigue syndrome is caused by an infected or suspected covid-19

Differentiation of syndrome: Dampness accumulated throughout three burners;

Both of Qi and Yin deficiency.

Herbal powders prescription: Xiaochaihu and Sanren Decoction variation

Jiangbanxia15, Chaihu10, Huangqin10, Ganjiang10,

Xingren10, Huoxiang10, Sharen10, Baidoukou10,

Huashi15, Tongcao10, Fuling10, Gancao5

Above herbal powders are mixed following with prescription, $6 \mathrm{~g}$ per time with some warm water, twice daily.

\section{Result and analysis:}

After she is given prescribed herbal powders in two weeks, she has been much better for her headache and general aching, so patent herbs Xiaochahu Pills and Buzhongyiqi pills are given until she fells much energy increased and better for live constitution.

\section{Case 5, Infection with the Man reproductive organs}

Mr. H, 28 years old of PHD student, requires for his fever with dull aching at low abdominal and groins for a week; he has a fever 37.4, dull pain at low abdomen, distension, pain at groins and testicles. He says he has been double carefully for himself since some classmates suffer from Covid-19, he confuses whether these symptoms can indicate he is infected or not? Whether does he need a treatment? (Tongue photo at left)

After my further requirements, he confirms he feels some abdominal discomfort while he is running, some sore and pressed pain at left of his scrotum. Red tongue with white greasy coating, thick at the base. Due to worry to Covid-19, he dislikes to go to hospital for doing PCR test.

Diagnosis: 1 Suspected Covid-19

\section{Seminal Vesiculitis}

Differentiation of syndrome: Heat and dampness accumulated within and spread throughout three burners

Herbal Prescription: Huopuxialing variated with Simiao decoction

Huanglian10, Houpo10, Huangbai10, Cangzhu10;

Chuanniuxi15, Yiyiren30, Zexie10, Chishao10,

Danshen10, Jiangbanxia15, Fuling10, Gancao5.

Above herbs are decocted as an herbal juice, twice daily, a dose for a day.

\section{Result and analysis:}


After he take herbal decoction form for a week, pain at abdomen, groins and scrotum are less, temperature has gone down to normal and thinkgreasy coating less as well. He is given Simiao Pills and Chaihushugan pill for taking until all of symptoms are completely controlled. (photo at right)

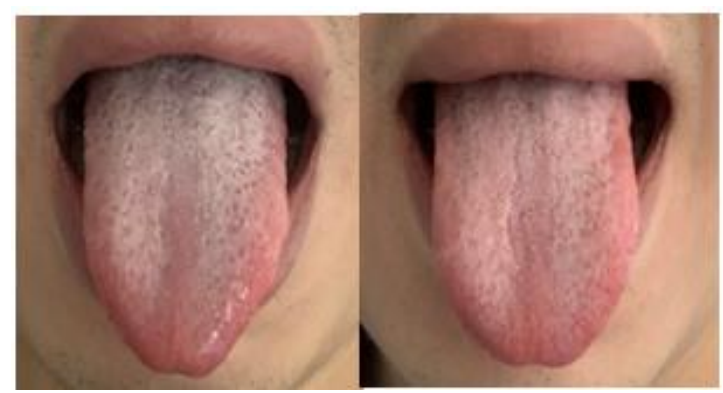

Image 5

According to reports in China, many young men who are infected as Covid-19 can complicate with inflammation at reproductive organs, and can leave them as a post-syndromes there after they are healed their infection at lungs from Convid-19. These inflammation on the reproductive organs can gradually recover, but leave some adhesion and scars in the testicle(s) or prostate (s) which may disturb stored sperm there for producing malformation and nonliquefaction of semen to cause an infertility? If this result appearance, this will influence the latter half live for this young man. So I add Danshen, Chishao in the main proscription, and use Simiao with Chaihushugan pills for preventing adhesion and inflammation in the reproductive organs after completely eliminating coronavirus in whole body with the main prescription.

\section{Discussion:}

These few of cases are recognized and treated by me as post-syndromes caused along Covid-19. Actually, after the covid-19 is spreading throughout world for more than a year, the post-syndrome caused or related with it has been many more. Many reports can be read from international Medias and medical journals in which are recognized as: functional inferior or fibrosis on lungs, on Liver or Kidney; damage to the central or peripheral nerve system, and cardiovascular system as well. Following in the time, more and more post-syndromes after such more patients are infected will appear which will give more refractory cases on post-syndromes of Covid-19. They also are opportunity and challenge to TCM. I summarize as below:

TCM is a superiority treating system to virus infection. A good designed prescription of Chinese herbal medicine can effectively control virus infection, restrain inflammation reaction, regulate immune function and promote self-healing capability, so it can possess a comprehensive treating effect with a multiple triggers to Covid-19 which can be proved by laboratory research. $(7,8)$

TCM is an effective treating system to many kinds of post-syndromes which are caused or related with Covid-19; it should be an excellent choice to the post-syndromes of Covid-19.

For patients with infectious Covid-19, special on these who have not completely healed after intensive treatment, TCM should be given as soon as possible for increasing their healing level, promoting their constitution and preventing serious complications' occurrence.

4Psychiatric disorders are the biggest secondary diseases accompanying along a pandemic disease spreading which is the commonest postsyndrome with this Covid-19. When we reopen a normal clinic with whole system of TCM treatments can be used, we will be more increasing our effective rate and cure rate if we can use acupuncture.

\section{Conclusion:}

Good designed TCM prescription should be the suitable treatment to Postsyndromes of Covid-19.

\section{Reference:}

1. Internet: World Health Organization. (2021). Coronavirus disease 2019 (COVID-19) pandemic.

2. GOV.UK. (2021). Coronavirus (COVID-19) in the UK.

3. Xia WG, An CQ, Zhang BL et al. (2020). Clinical study on integrative medicine treat Novel corona pneumonia with 34 cases study; Journal of Chinese Medicine. 5, 1 (Chinese ) R563.1.

4. China's National Health Commission, Novel Coronavirus Treatment Guideline- $7^{\text {th }}$ Edition from UCLA Health Campus Initiative (HCI) etc.

5. D Jiang Diagnosis and Management of TCM on Infected or Suspected Patients with Covid------48 Cases study in UK, Annals of Clinical Cases 2021 - Medtext Publications. 2692-7993.

6. Professional standard authority in BAcC, Coronavirus information, Health information.

7. Ma LQ, Pan CS, Han JY et al. (2014). Posttreatment with MaXing-Shi-Gan-Tang, a Chinese medicine formula, ameliorates lipopolysaccharide-induced lung microvessel hyperpermeability and inflammatory reaction in rat. Microcirculation. 21 (7): 649.

8. Yu LP, Wang XK and Luo JB. (2011). Determination of Ephedrine Hydrochloride, Pseudoephedrine Hydrochloride and Methylephedrine Hydrochloride in Maxingshigan Decoction by CE, (Chinese) Materia metica. 34(4): 620.

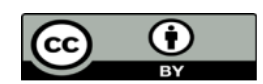

This work is licensed under Creative Commons Attribution 4.0 License

\section{To Submit Your Article Click Here: Submit Manuscript}

DOI: $10.31579 / 2768-0487 / 046$
Ready to submit your research? Choose Auctores and benefit from:

$>$ fast, convenient online submission

$>$ rigorous peer review by experienced research in your field

$>$ rapid publication on acceptance

$>$ authors retain copyrights

$>$ unique DOI for all articles

$>$ immediate, unrestricted online access

At Auctores, research is always in progress.

Learn more auctoresonline.org/journals/journal-of-clinical-andlaboratory-research 